\title{
A RELATION ON SPIN BUNDLE GERBES AND MAYER'S DIRAC OPERATORS
}

\author{
ATSUSHI TOMODA \\ Department of Mathematics, Keio University, \\ 3-14-1 Hiyoshi, Kohoku-ku, Yokohama, Japan \\ E-mail:ton@math.keio.ac.jp
}

\begin{abstract}
Exploiting the notion of bundle gerbe due to Murray, Murray and Singer constructed a generalization of Dirac operators for possibly non-spin manifolds. We shall provide an alternative proof for their index formula, and clarify the relation between a generalized Dirac operators due to Mayer and their operators. Furthermore, we determine the twisted Chern character of some bundle gerbe modules.
\end{abstract}

\section{Introduction}

Murray and Singer introduced the Dirac operators associated with the spin bundle gerbe modules, which are, roughly speaking, roots of complex vector bundles, by the use of the spin bundle gerbes.

The purpose of this paper is to describe the index formula of the Dirac operators associated with spin bundle gerbe modules from a different point of view. Mayer introduced Mayer's Dirac operator associated with a $G(2 l, n, 2)$-structure $\sigma$. We also relate their operators with the Mayer's Dirac operators and describe the twisted Chern character for some bundle gerbe modules explicitly:

Theorem 0.1. Let $\sigma$ be a $G(2 l, n, 2)$-structure of $X$ induced by a real vector bundle $E$ with $W_{3}(T X \oplus E)=0$ and let $F$ be the associated line bundle of $\sigma$. Then we have

$$
\operatorname{ch}^{\tau}\left(W_{\sigma}\right)=2^{[n / 2]} e^{d / 2} \prod_{i=1}^{[n / 2]} \cosh \left(x_{i} / 2\right),
$$

where $d=c_{1}(F)$ and $p(E)=\prod_{i}\left(1+x_{i}^{2}\right)$. 


\section{Bundle gerbes}

\subsection{Bundle gerbes}

We shall give an exposition of the bundle gerbes. We refer to Murray ${ }^{5}$.

Let $X$ be a compact oriented smooth manifold and let $\pi: Y \rightarrow X$ be a fiber bundle. Then we can consider the fiber product $Y \times_{\pi} Y$ with itself. For simplicity, we denote this by $Y^{[2]}$.

Definition 1.1. Let $X$ and $Y$ be as above and let $L \rightarrow Y^{[2]}$ be a hermitian line bundle. A triple $(X, Y, L)$ is said to be a bundle gerbe over $X$ if $L$ is equipped with a product which is an isomorphism

$$
L_{\left(y_{1}, y_{2}\right)} \otimes L_{\left(y_{2}, y_{3}\right)} \stackrel{\cong}{\longrightarrow} L_{\left(y_{1}, y_{3}\right)} \quad \text { for every }\left(y_{1}, y_{2}\right),\left(y_{2}, y_{3}\right) \in Y^{[2]},
$$

and which is associative.

As line bundles have Euler classes, bundle gerbes have a kind of characteristic classes:

Definition 1.2. Fix a good cover $\left\{U_{\alpha}\right\}$ of $X$ and local sections $\left\{s_{\alpha}\right.$ : $\left.U_{\alpha} \rightarrow P\right\}$. Then we obtain line bundles $L_{\alpha \beta}=\left(s_{\alpha}, s_{\beta}\right)^{*} L$ over $U_{\alpha \beta}$. A choice of sections $z_{\alpha \beta} \in \Gamma\left(L_{\alpha \beta}\right)$ and the induced product gives a unique $\left\{\varepsilon_{\alpha \beta \gamma}: U_{\alpha \beta \gamma} \rightarrow U(1)\right\}$ such that

$$
L_{\alpha \beta} \otimes L_{\beta \gamma} \rightarrow L_{\alpha \gamma} ; z_{\alpha \beta} \otimes z_{\beta \gamma} \mapsto \varepsilon_{\alpha \beta \gamma} z_{\alpha \gamma} .
$$

Then $\left\{\varepsilon_{\alpha \beta \gamma}\right\}$ gives a cohomology class $\left[\left\{\varepsilon_{\alpha \beta \gamma}\right\}\right] \in \check{H}^{2}(X ; U(1)) \cong \check{H}^{3}(X ; \mathbb{Z})$ and we denote this by $\operatorname{dd}(X, Y, L)$ and we call this the Dixmier-Douady class for the bundle gerbe $(X, Y, L)$.

Remark 1.1. The Dixmier-Douady class $\operatorname{dd}(X, Y, L)$ of $(X, Y, L)$ does not depend on the choice of open covering of $X$ and local sections $\left\{s_{\alpha}\right\}$ and $\left\{z_{\alpha \beta}\right\}$.

Example 1.1. Consider a principal $S O(n)$-bundle $P$ and a central extension:

$$
1 \rightarrow \mathbb{Z}_{2} \rightarrow \operatorname{Spin}(n) \stackrel{p}{\rightarrow} S O(n) \rightarrow 1
$$

We define a $\mathbb{Z}_{2}$-bundle $Q$ over $P^{[2]} \rightarrow X$ by

$$
Q=\left\{\left(\left(y_{1}, y_{2}\right), \alpha\right) \in P^{[2]} \times \operatorname{Spin}(n) \mid y_{1} p(\alpha)=y_{2}\right\}
$$

so we obtain the complex line bundle $L \rightarrow P^{[2]}$ associated with $Q$. Then the triple $(X, P, L)$ is a bundle gerbe over $X$ and we call this the spin bundle 
gerbe of $P$. Especially every oriented closed $n$-dimensional manifold with a Riemannian metric yields a $S O(n)$-frame bundle $P$ of $T X$ and hence a spin bundle gerbe $(X, P, L)$ of $P$. We simply call this the spin bundle gerbe of $X$.

We note that the Dixmier-Douady class $\operatorname{dd}(X, P, L)$ of the spin bundle gerbe $(X, P, L)$ of $X$ coincides with the third integral Stiefel-Whitney class $W_{3}(X)$.

In the subsequent discussion we deal with only spin bundle gerbes.

\subsection{Bundle gerbe modules and the twisted Chern character}

We introduce the notion of bundle gerbe modules. This is introduced in Bouwknegt, Carey, Mathai, Murray, and Stevenson ${ }^{3}$.

Definition 1.3. Let $(X, P, L)$ be a spin bundle gerbe. Then a hermitian vector bundle $W$ over $P$ is called a bundle gerbe module for $(X, P, L)$ if it is endowed with the multiplication of $L$ :

$$
L_{\left(y_{1}, y_{2}\right)} \otimes W_{y_{2}} \cong W_{y_{1}} \quad \text { for every }\left(y_{1}, y_{2}\right) \in P^{[2]}
$$

which satisfies the commutative diagram:

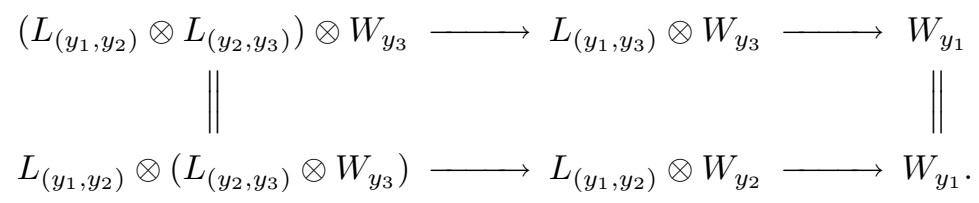

We denotes by $\operatorname{Mod}(X, P, L)$ the isomorphism classes of bundle gerbe modules for $(X, P, L)$.

$\operatorname{Spin}(n)$ is naturally included in $L$. Hence $\operatorname{Spin}(n)$ acts on a bundle gerbe module $W$. The action of $\mathbb{Z}_{2} \subset \operatorname{Spin}(n)$ on each fiber $W_{y}$ is multiplication by \pm 1 . Consider two bundle gerbe modules $V$ and $W$. Then $\operatorname{Spin}(n)$ acts on $V \otimes W$ and the action induces $S O(n)$-action. Moreover, it gives rise to a complex vector bundle $E$ over $X$ satisfying $\pi^{*} E=V \otimes W$. We denote this by $(V \otimes W)_{0}$.

Let $(X, P, L)$ be the spin bundle gerbe for $X$. Then we have a canonical flat connection $\nabla$ on $L$ induced by $\mathbb{Z}_{2}$-bundle $Q . \quad \nabla$ is a bundle gerbe connection. That is, the product of $L$ preserves the connection.

Definition 1.4. Let $W \rightarrow P$ be a bundle gerbe module for $(X, P, L)$. Then a hermitian connection $\nabla^{W}$ on $W$ is called a bundle gerbe module 
connection on $W$ compatible with $(X, P, L, \nabla)$ if the multiplication

$$
\varphi: L \otimes \pi_{1}^{*} W \stackrel{\cong}{\rightrightarrows} \pi_{2}^{*} W
$$

preserves the connections, where $L \otimes \pi_{1}^{*} W\left(\pi_{2}^{*} W\right.$ resp. $)$ is endowed with the induced connection $\nabla \otimes 1+1 \otimes \pi_{1}^{*} \nabla^{W}\left(\pi_{2}^{*} \nabla^{W}\right.$ resp. $)$. Here $\pi_{i}: Y^{[2]} \rightarrow Y$ denotes the $i$-th element forgetting map for $i=1,2$. If there is no confusion, we simply call $\nabla^{W}$ a bundle gerbe module connection on $W$.

Let $\nabla^{W}$ be a bundle gerbe module connection. Then we have

$$
1 \otimes \pi_{1}^{*} F\left(\nabla^{W}\right)^{k}=\varphi \circ \pi_{2}^{*} F\left(\nabla^{W}\right)^{k} \circ \varphi^{-1} \text { for every } k>0 .
$$

So every invariant polynomial $P$ defines $P\left(F\left(\nabla^{W}\right)\right) \in \Omega^{2 *}(P)$ and $\left(\pi_{1}^{*}-\right.$ $\left.\pi_{2}^{*}\right) P\left(F\left(\nabla^{W}\right)\right)=0$. Therefore we obtain $[\eta] \in H^{2 *}(X)$ such that $\pi^{*} \eta=$ $P\left(F\left(\nabla^{W}\right)\right)$. The cohomology class $[\eta]$ does not depend on a choice of bundle gerbe module connections $\nabla^{W}$. Especially tr is invariant polynomial and there is unique $\left[\eta_{k}\right] \in H^{2 k}(X)$ such that

$$
\pi^{*}\left[\eta_{k}\right]=\operatorname{tr}\left(\left(\frac{-1}{2 \pi i}\left(F\left(\nabla^{W}\right)\right)\right)^{k}\right)
$$

Definition 1.5. Analogously to Chern character of complex vector bundles, we define the twisted Chern character $\operatorname{ch}^{\tau}(W)$ of a bundle gerbe module $W$ by

$$
\operatorname{ch}^{\tau}(W)=\left[\eta_{0}\right]+\left[\eta_{1}\right]+\cdots+\left[\eta_{k}\right]+\cdots \in H^{2 *}(X ; \mathbb{R}) .
$$

\section{Dirac operators associated with bundle gerbe modules}

Let $X$ be a $2 l$-dimensional smooth oriented closed manifold and let $(X, P, L)$ be the lifting bundle gerbe of $X$. In this section we shall introduce the Dirac operator associated with a bundle gerbe module for a probably non-spin manifold.

Definition 2.1. Let $(X, P, L)$ be as above. Then we define $\mathbb{S}(P)$ as

$$
\mathbb{S}(P)=P \times \mathbb{S}_{2 l} \text {, where } \mathbb{S}_{2 l} \text { denotes the spinor space of } \mathbb{R}^{2 l} \text {. }
$$

It is easy to see that $\mathbb{S}(P) \rightarrow P$ is a bundle gerbe module for $(X, P, L)$, and we call $\mathbb{S}(P)$ the spinor bundle gerbe module of $P$.

Endowed with some Riemannian metric $g, X$ has the Levi-Civita connection $\nabla^{L C}$ on $T X$. The connection $\nabla^{L C}$ induces a connection 1-form 
$\omega \in \Omega^{1}(P ; \mathfrak{s o}(2 l))$ on $P$. Here we note that there is the natural isomorphism $\varphi_{*}: \mathfrak{s p i n}(2 l) \stackrel{\cong}{\mathfrak{s}}(2 l)$ induced by $\varphi: \operatorname{Spin}(2 l) \rightarrow S O(2 l)$. The spinor representation $\rho: \operatorname{Spin}(2 l) \rightarrow \operatorname{Aut}\left(\mathbb{S}_{2 l}\right)$ gives rise to $\rho_{*}:$ $\mathfrak{s p i n}(2 l) \rightarrow \operatorname{End}_{+}\left(\mathbb{S}_{2 l}\right)$. The composition $\rho_{*} \circ \varphi_{*}^{-1}: \mathfrak{s o}(2 l) \rightarrow \operatorname{End}_{+}\left(\mathbb{S}_{2 l}\right)$ defines $\widetilde{\omega} \in \Omega^{1}\left(P ; \operatorname{End}_{+} \mathbb{S}(P)\right)$ by $\widetilde{\omega}=\rho_{*} \circ \varphi_{*}^{-1}(\omega)$. We define a canonical connection $\widetilde{\nabla}$ on $\mathbb{S}(P)$ by $\widetilde{\nabla}=d+\widetilde{\omega}$.

We can easily show that $\widetilde{\nabla}$ is a bundle gerbe module connection on $\mathbb{S}(P)$.

Next, we shall define Clifford multiplication $\widetilde{c}$ on $P$. The connection 1-form $\omega \in \Omega^{1}\left(P ;\right.$ End $\left._{-}(\mathbb{S}(P))\right)$ induces

$$
T^{*} P \rightarrow \pi^{*} T^{*} X
$$

Moreover,

$$
P \times \mathbb{R}^{2 l} \times \mathbb{S}_{2 l} \rightarrow P \times \mathbb{S}_{2 l} ;(y, v, \xi) \mapsto(y, v \xi)
$$

defines $c: \pi^{*} T X \rightarrow$ End $_{-}(\mathbb{S}(P))$ since $P \times_{S O(2 l)} \mathbb{R}^{2 l}$ is isomorphic to $T X$. So we have

$$
\widetilde{c}: T^{*} P \rightarrow \pi^{*} T^{*} X \stackrel{\cong}{\longrightarrow} \pi^{*} T X \stackrel{c}{\rightarrow} \operatorname{End}\left(\mathbb{S}^{ \pm}(P), \mathbb{S}^{\mp}(P)\right) .
$$

We have prepared some notions so far. Now we define the Dirac operator $\not D_{W}$ associated with a bundle gerbe module $W$.

Choose and fix a bundle gerbe module $W$ for $(X, P, L)$ and an arbitrary bundle gerbe module connection $\nabla^{A}$ compatible with $\left(X, P, L, \nabla^{L}\right)$. Then we obtain $\bar{D}_{W, A}$ as the composition:

$$
\bar{D}_{W, A}=(\widetilde{c} \otimes \mathrm{Id}) \circ\left(\widetilde{\nabla} \otimes 1+1 \otimes \nabla^{A}\right) .
$$

We have to note that $\bar{D}_{W, A}$ is a first order non-elliptic differential operator from $\mathbb{S}^{+}(P) \otimes W$ to $\mathbb{S}^{-}(P) \otimes W$ over $P$ because the symbol $\sigma\left(\bar{D}_{W, A}\right)$ vanishes in direction of the fiber of $P$. However, $\bar{D}_{W, A}$ is $\operatorname{Spin}(2 l)$-equivariant operator and it gives rise to an elliptic operator

$$
\not D_{W, A}: \Gamma\left(X,\left(\mathbb{S}^{+}(P) \otimes W\right)_{0}\right) \rightarrow \Gamma\left(X,\left(\mathbb{S}^{-}(P) \otimes W\right)_{0}\right)
$$

over $X$. We call this the Dirac operator associated with a bundle gerbe module $W$. The next theorem is due to Murray and Singer ${ }^{6}$ :

Theorem 2.1.

$$
\operatorname{Index}\left(\not D_{W, A}\right)=\int_{X} \widehat{\mathcal{A}}(X) \operatorname{ch}^{\tau}(W)
$$


They proved this theorem by the use of the method of Getzler, Berline and Vergne $^{2}$. Here we prove in an alternative way.

Proof. The property of the twisted Chern character implies

$$
\operatorname{ch}\left(\left(\mathbb{S}^{ \pm} \otimes W\right)_{0}\right)=\operatorname{ch}^{\tau}\left(\mathbb{S}^{ \pm}\right) \operatorname{ch}^{\tau}(W) .
$$

Atiyah-Singer index theorem ${ }^{1}$ says:

$$
\begin{aligned}
\operatorname{Index}\left(\not D_{W, A}\right) & =(-1)^{l} \frac{\operatorname{ch}\left(\left(\mathbb{S}^{+} \otimes W\right)_{0}-\left(\mathbb{S}^{-} \otimes W\right)_{0}\right) \operatorname{td}(T X \otimes \mathbb{C})}{e(X)}[X] \\
& =(-1)^{l} \frac{\left(\prod_{i=1}^{l} e^{x_{i} / 2}-e^{-x_{i} / 2}\right)}{x_{1} \cdots x_{l}} \prod_{i=1}^{l} \frac{x_{i}}{1-e^{-x_{i}}} \frac{-x_{i}}{1-e^{x_{i}}} \operatorname{ch}^{\tau}(W)[X] \\
& =\widehat{A}(X) \operatorname{ch}^{\tau}(W)[X] .
\end{aligned}
$$

The second equality is derived from (1) and the next theorem.

The next theorem is essential.

Theorem 2.2. For every $S O(2 l)$-bundle $P \rightarrow X$, we have the spinor bundle gerbe module $\mathbb{S}^{ \pm}(P)$, which is a bundle gerbe module for $(X, P, L)$. Then we have

$$
\operatorname{ch}^{\tau}\left(\mathbb{S}^{+}(P)-\mathbb{S}^{-}(P)\right)=\prod_{i=1}^{l}\left(e^{x_{i} / 2}-e^{-x_{i} / 2}\right)
$$

where the associated rank $m$ vector bundle $E=P \times_{S O(2 l)} \mathbb{R}^{2 l}$ virtually splits into $l$ complex line bundles $L_{1} \oplus \cdots \oplus L_{l}$ and we put $c_{1}\left(L_{i}\right)=x_{i}$ for every $i$.

Proof. Consider a vector bundle $E=P \times_{S O(2 l)} \mathbb{R}^{2 l} \rightarrow X$. Then we have $E=L_{1} \oplus \cdots L_{l}$ virtually. Since $w_{2}(E \oplus E)=0$, we have a complex vector bundle $\mathbb{S}(E \oplus E)$ over $X$, and

$$
\mathbb{S}^{+}(E \oplus E)-\mathbb{S}^{-}(E \oplus E)=\bigotimes_{i=1}^{l}\left(\mathbb{S}^{+}\left(L_{i} \oplus L_{i}\right)-\mathbb{S}^{-}\left(L_{i} \oplus L_{i}\right)\right)
$$

Easy calculation gives us the explicit description of Chern character:

$$
\operatorname{ch}\left(\mathbb{S}^{+}\left(L_{i} \oplus L_{i}\right)-\mathbb{S}^{-}\left(L_{i} \oplus L_{i}\right)\right)=e^{x_{i}}+e^{-x_{i}}-2 \text { for } i=1, \ldots, l,
$$


where $x_{i}=c_{1}\left(L_{i}\right)$. So we have

$$
\begin{aligned}
\operatorname{ch}\left(\mathbb{S}^{+}(E \oplus E)-\mathbb{S}^{-}(E \oplus E)\right) & =\prod_{i=1}^{l}\left(e^{x_{i}}+e^{-x_{i}}-2\right) \\
& =\left(\prod_{i=1}^{l}\left(e^{x_{i} / 2}-e^{-x_{i} / 2}\right)\right)^{2} .
\end{aligned}
$$

On the other hand,

$$
\operatorname{ch}^{\tau}\left(\mathbb{S}^{+}\left(P_{E}\right)-\mathbb{S}^{-}\left(P_{E}\right)\right)^{2}=\operatorname{ch}\left(\mathbb{S}^{+}(E \oplus E)-\mathbb{S}^{-}(E \oplus E)\right) .
$$

By considering the universal bundle $E S O(2 l) \rightarrow B S O(2 l)$ and the spin bundle gerbe $(B S O(2 l), E S O(2 l), \widetilde{L})$ of $E S O(2 l)$, we obtain

$$
\operatorname{ch}^{\tau}\left(\mathbb{S}^{+}(E S O(2 l))-\mathbb{S}^{-}(E S O(2 l))\right)= \pm \prod_{i=1}^{l}\left(e^{x_{i} / 2}-e^{-x_{i} / 2}\right)
$$

since the ring $H^{*}(B S O(2 l) ; \mathbb{R})$ has no zero divisor. So far, the equation holds up to signature. For every $S O(2 l)$-bundle $P \rightarrow X$, there exists $f$ : $X \rightarrow B S O(2 l)$ satisfying $f^{*} E S O(2 l) \cong P$, and $\mathbb{S}^{ \pm}(P)$ comes from bundle gerbe module $\mathbb{S}^{ \pm}(E S O(2 l))$ for $(B S O(2 l), E S O(2 l), \widetilde{L})$. Strictly speaking,

$$
\mathbb{S}^{ \pm}(P) \cong \bar{f}^{*}\left(\mathbb{S}^{ \pm}(E S O(2 l))\right.
$$

where $\bar{f}: P \rightarrow E S O(2 l)$ is a bundle map covering $f: X \rightarrow B S O(2 l)$. Twisted Chern character has the naturality. Hence the equation (2) is valid for every $S O(2 l)$-bundle $P \rightarrow X$.

Since the signature is universally determined, we can see which is correct by a non-trivial example. For a $S O(2 l)$-bundle which admits spin structure, the signature is positive. Hence we can determine the signature in (2).

We shall mention $\operatorname{spin}^{c}$ structures in the view point of bundle gerbe. A $\operatorname{spin}^{c}$ structure is the unit vector bundle of a bundle gerbe module $G$ with rank 1. Then $\operatorname{ch}^{\tau}(G)=e^{d / 2}$, where we set $d=c_{1}\left(\left(G^{\otimes 2}\right)_{0}\right)$. Especially a spin structure is one associated with a $\mathbb{Z}_{2}$-bundle over $P$ and $\left(G^{\otimes 2}\right)_{0}=\underline{\mathbb{C}}$. Therefore $\operatorname{ch}^{\tau}(G)=1$.

Mayer $^{4}$ defined the Dirac operator associated with some principal bundle. First, we recall the construction. Consider

$$
S O(m) \times S O(n) \times S O(2) \subset S O(m+n+2)
$$

and $\lambda: \operatorname{Spin}(m+n+2) \rightarrow S O(m+n+2)$. Then we can identify $\lambda^{-1}(S O(m) \times S O(n) \times S O(2))$ with $(\operatorname{Spin}(m) \times \operatorname{Spin}(n) \times \operatorname{Spin}(2)) / \mathbb{Z}_{2} \oplus \mathbb{Z}_{2}$, 
where $\mathbb{Z}_{2} \oplus \mathbb{Z}_{2}$ is generated by $\{(-1,-1,+1),(+1,-1,-1)\}$. We denote this by $G(m, n, 2)$.

Definition 2.2. Let $\left\{g_{\alpha \beta}\right\} \in \check{C}^{2}(X, S O(m))$ denote the Cech cocycle defining the frame bundle $P$ of $T X$. Then $\left\{\left[\widehat{g}_{\alpha \beta}, \widehat{h}_{\alpha \beta}, \widehat{z}_{\alpha \beta}\right]\right\} \in \check{C}^{2}(X, G(m, n, 2))$ is called a $G(m, n, 2)$-structure if

$$
\lambda\left(\widehat{g}_{\alpha \beta}\right)=g_{\alpha \beta} \text { and } \delta\left\{\left[\widehat{g}_{\alpha \beta}, \widehat{h}_{\alpha \beta}, \widehat{z}_{\alpha \beta}\right]\right\}=1 .
$$

We have the complex spinor representation $\rho^{c}: G(2 l, n, 2) \rightarrow \operatorname{Aut}\left(\mathbb{S}_{2 l} \otimes\right.$ $\left.\mathbb{S}_{n}\right)$. Given a $G(2 l, n, 2)$-structure $\sigma=\left\{\left[\widehat{g}_{\alpha \beta}, \widehat{h}_{\alpha \beta}, \widehat{z}_{\alpha \beta}\right]\right\}$ over $2 l$-dimensional manifold $X$, we obtain a spinor bundle $\mathbb{S}_{\sigma}$ associated with it and the Dirac operator $\not_{\sigma}: \Gamma\left(\mathbb{S}_{\sigma}^{+}\right) \rightarrow \Gamma\left(\mathbb{S}_{\sigma}^{-}\right)$. We call this Mayer's Dirac operator.

If a real vector bundle $E \rightarrow X$ with rank $n$ satisfies $W_{3}(T X \oplus E)=$ 0 , then we have a $G(2 l, n, 2)$-structure $\sigma=\left\{\left[\widehat{g}_{\alpha \beta}, \widehat{h}_{\alpha \beta}, \widehat{z}_{\alpha \beta}\right]\right\}$ such that $\left\{\lambda\left(\widehat{h}_{\alpha \beta}\right)\right\}$ defines $E$ and $\left\{\lambda\left(\widehat{z}_{\alpha \beta}\right)\right\}$ defines a complex line bundle $F$ which satisfies $w_{2}(T X)+w_{2}(E)=w_{2}(F)$. Mayer proved:

Theorem 2.3. Let $X, E$, and $F$ be as above. Then we have

$$
\operatorname{Index} \not D_{\sigma}=2^{[n / 2]} \int_{X} e^{d / 2} \widehat{A}(X) \prod_{i=1} \cosh \left(x_{i} / 2\right),
$$

where $d=c_{1}(F)$ and $p(E)=\prod_{i}\left(1+x_{i}^{2}\right)$.

Here we shall introduce twisted unitary structure of $X$. We define $U^{\tau}(n)$ as $U^{\tau}(n)=\operatorname{Spin}(2 l) \times_{ \pm 1} U(n)$. Then $U(n) \hookrightarrow U^{\tau}(n)$ is a normal subgroup. The twisted unitary structures $U^{\tau}(n)(X)$ of $X$ are defined by

$$
U^{\tau}(n)(X)=\left\{\widetilde{P} \rightarrow X: U^{\tau}(n) \text {-bundle } \mid \widetilde{P} / U(n) \text { is isomorphic to } P\right\} .
$$

Theorem 2.4. We obtain the isomorphism

$$
U^{\tau}(n)(X) \cong \operatorname{Mod}_{n}(X, P, L) ; \widetilde{P} \mapsto \widetilde{P} \times_{U(n)} \mathbb{C}^{n}=W_{\widetilde{P}} .
$$

Proof. For every $W \in \operatorname{Mod}_{n}(X, P, L)$ a $U(n)$-bundle $\widetilde{P}_{W}$, which consists of unitary frames of $W$, defines a twisted unitary structure. This is the inverse.

The homomorphism $\operatorname{Spin}(n) \times_{\mathbb{Z}_{2}} \operatorname{Spin}(2) \rightarrow U\left(2^{[n / 2]}\right)$ defines a homomorphism $G(m, n, 2) \rightarrow U^{\tau}\left(2^{[n / 2]}\right)$. Then we obtain 
Theorem 2.5. Every $G(2 l, n, 2)$-structure $\sigma$ gives rise to a bundle gerbe module $W_{\sigma}$. Moreover $\operatorname{Index}\left(\not D_{\sigma}\right)=\operatorname{Index}\left(\not D_{W_{\sigma}}\right)$ for Mayer's Dirac operator $D_{\sigma}$.

Let $\sigma$ be a $G(2 l, n, 2)$-structure over $X$. We denote the associated real vector bundle by $E$ and the associated complex line bundle by $F$. Then we have a spin bundle gerbe $\left(X, P_{E}, L_{E}\right)$ of $E$, and a bundle gerbe module $\mathbb{S}\left(P_{E}\right)$ for $\left(X, P_{E}, L_{E}\right)$, where $P_{E}$ denotes the $S O(n)$-frame bundle of $E$. Like as the proof of Theorem2.2, we have

$$
\operatorname{ch}^{\tau}\left(\mathbb{S}\left(P_{E}\right)\right)=\prod_{i=1}^{[n / 2]}\left(e^{x_{i} / 2}+e^{-x_{i} / 2}\right)=2^{[n / 2]} \prod_{i} \cosh \left(x_{i} / 2\right),
$$

where $p(E)=\prod_{i}\left(1+x_{i}^{2}\right)$. We have to remark that $\mathbb{S}\left(P_{E}\right)$ is a bundle gerbe module not for $(X, P, L)$ but for $\left(X, P_{E}, L_{E}\right)$.

We shall consider another spin bundle gerbe $\left(X, P \times_{\pi} P_{E}, L \otimes L_{E}\right)$. It is easy to see there is a bundle gerbe module $G$ for this with rank 1 satisfying $\left(G^{\otimes 2}\right)_{0}=F$ since $W_{3}(T X)+W_{3}(E)=0$. Then $G$ induces an isomorphism $G \otimes: \operatorname{Mod}\left(X, P_{E}, L_{E}\right) \rightarrow \operatorname{Mod}(X, P, L)$. We will prove that $G \otimes$ maps $\mathbb{S}\left(P_{E}\right)$ to the bundle gerbe module $W_{\sigma}$ for $(X, P, L)$ and that

$$
\operatorname{ch}^{\tau}\left(W_{\sigma}\right)=e^{d / 2} \operatorname{ch}^{\tau}(\mathbb{S}(E))=2^{[n / 2]} e^{d / 2} \prod_{i} \cosh \left(x_{i} / 2\right) .
$$

Consider the next diagram:

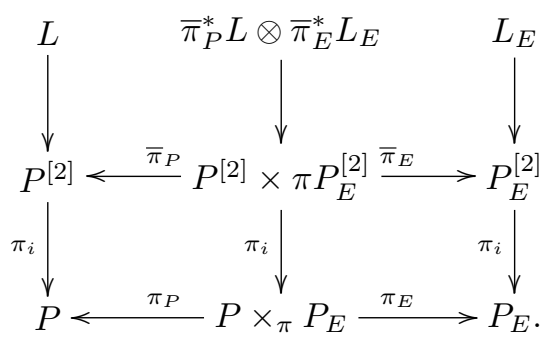

It is easy to see $\pi_{1}^{*} G \otimes \pi_{2}^{*} G^{*} \cong \bar{\pi}^{*} L \otimes \bar{\pi}_{E}^{*} L_{E}$. We define $Q_{y}$ and $Q_{z}$ by

$$
\begin{aligned}
& Q_{y}=\left\{\left(\left(y_{1}, y_{2}\right),\left(z_{1}, z_{2}\right)\right) \in P^{[2]} \times_{\pi} P_{E}^{[2]} \mid y_{1}=y_{2}\right\}, \\
& Q_{z}=\left\{\left(\left(y_{1}, y_{2}\right),\left(z_{1}, z_{2}\right)\right) \in P^{[2]} \times_{\pi} P_{E}^{[2]} \mid z_{1}=z_{2}\right\} .
\end{aligned}
$$


Then, $\pi_{1}^{*} G \otimes \pi_{2}^{*} G^{*}$ is isomorphic to $\bar{\pi}_{E}^{*} L_{E}$ over $Q_{y}$, and to $\bar{\pi}^{*} L$ over $Q_{z}$. We obtain the isomorphisms:

$$
\begin{aligned}
\pi_{2}^{*}\left(G \otimes \pi_{E}^{*} \mathbb{S}\left(P_{E}\right)\right) & =\pi_{2}^{*}(G) \otimes \pi_{P}^{*} \pi_{E}^{*} \mathbb{S}\left(P_{E}\right)=\pi_{2}^{*} G \otimes \bar{\pi}_{E}^{*} \pi_{2}^{*} \mathbb{S}\left(P_{E}\right) \\
& =\pi_{2}^{*} G \otimes \bar{\pi}_{E}^{*}\left(L_{E} \times \pi_{1}^{*} \mathbb{S}\left(P_{E}\right)\right) \\
& =\pi_{2}^{*} G \otimes \bar{\pi}_{E}^{*} L_{E} \otimes \pi_{1}^{*} \pi_{E}^{*} \mathbb{S}\left(P_{E}\right) \\
& =\pi_{1}^{*} G \otimes \pi_{1}^{*} \pi_{E}^{*} \mathbb{S}\left(P_{E}\right)=\pi_{1}^{*}\left(G \otimes \pi_{E}^{*} \mathbb{S}\left(P_{E}\right)\right) \text { over } Q_{y}
\end{aligned}
$$

and

$$
\begin{aligned}
\bar{\pi}^{*} L \otimes \pi_{1}^{*}\left(G \otimes \pi_{E}^{*} \mathbb{S}\left(P_{E}\right)\right) & =\bar{\pi}^{*} L \otimes \pi_{1}^{*} G \otimes \pi_{1}^{*} \pi_{E}^{*} \mathbb{S}\left(P_{E}\right) \\
& =\left(\bar{\pi}^{*} L \otimes \pi_{E}^{*} L_{E}\right) \otimes \pi_{1}^{*} G \otimes \pi_{1}^{*} \pi_{E}^{*} \mathbb{S}\left(P_{E}\right) \\
& =\pi_{2}^{*} G \otimes \pi_{2}^{*} \pi_{E}^{*} \mathbb{S}\left(P_{E}\right) \\
& =\pi_{2}^{*}\left(E \otimes \pi_{E}^{*} \mathbb{S}\left(P_{E}\right)\right) \text { over } Q_{z} .
\end{aligned}
$$

The isomorphism (3) implies that there is a hermitian vector bundle $W^{\prime}$ over $P$ such that $\pi_{P}^{*} W^{\prime}$ is isomorphic to $G \otimes \pi_{E}^{*} \mathbb{S}\left(P_{E}\right)$, and (4) says that $W^{\prime}$ is a bundle gerbe module for $(X, P, L)$. It is easy to see that $W^{\prime}$ is isomorphic to $W_{\sigma}$ by the construction.

Moreover, these are isomorphisms with connections. Therefore, $\nabla^{G} \otimes$ $\pi_{E}^{*} \nabla^{\mathbb{S}\left(P_{E}\right)}$ induces a bundle gerbe module connection $\nabla^{W_{\sigma}}$ on $W_{\sigma}$ such that $\pi_{P}^{*} \nabla^{W_{\sigma}}=\nabla^{G} \otimes \pi_{E}^{*} \nabla^{\mathbb{S}\left(P_{E}\right)}$, and

$$
\operatorname{ch}^{\tau}\left(W_{\sigma}\right)=\operatorname{ch}^{\tau}(G) \operatorname{ch}^{\tau}\left(\mathbb{S}\left(P_{E}\right)\right)=e^{d / 2} \operatorname{ch}^{\tau}\left(\mathbb{S}\left(P_{E}\right)\right) .
$$

So we have proved Theorem.0.1.

\section{References}

1. M.F. Atiyah and I.M. Singer, The index of elliptic operators I. Ann. Math. 87 (1963), 484-530.

2. N. Berline, E. Getzler, and M. Vergne, Heat kernels and Dirac operators. Grundlehren Math. Wiss. 298 (1992), Springer-Verlag, New York.

3. P. Bouwknegt, A. Carey, V. Mathai, M.K. Murray, and D. Stevenson, Twisted $K$-theory and K-theory of bundle gerbes. Comm. Math. Phys. 228 (2002), no. $1,17-45$.

4. K.H. Mayer, Elliptische Differentialoperatoren und Ganzzahligkeitssätze für charakteristische Zahlen. Topology 4 (1965), 295-313.

5. M.K. Murray, Bundle gerbes. J. London. Math. Soc. (2) 54 (1996) no. 2, 403-416.

6. M.K. Murray and M.A. Singer, Gerbes, Clifford Modules and the Index Theorem. Ann. Global Anal. Geom. 26 (2004), 355-367. 